\title{
Quantum Dot Parameters Determination From Quantum-Efficiency Measurements
}

Antonio Luque,

Aleksandr Panchak, Iñigo Ramiro, Pablo Garcia-Linares, Alexandre Mellor, Elisa Antolín, Alexey Vlasov, Vyacheslav Andreev, and Antonio Martí

\begin{abstract}
The energy spectrum of the confined states of a quantum dot intermediate band (IB) solar cell is calculated with a simplified model. Two peaks are usually visible at the lowest energy side of the subbandgap quantum-efficiency spectrum in these solar cells. They can be attributed to photon absorption between welldefined states. As a consequence, the horizontal size of the quantum dots can be determined, and the conduction (valence) band offset is also determined if the valence (conduction) offset is known.
\end{abstract}

Index Terms-Energy states, optical pumping, photovoltaic cells, quantum dots.

\section{INTRODUCTION}

$\mathbf{T}$ HE intermediate band (IB) solar cell [1] is based on a material with bands or levels within the forbidden bandgap of a semiconductor. In it, two subbandgap photons can originate an electron-hole pair in the valence band (VB) and conduction band (CB) of the semiconductor, using the IB as a stepping stone. With this material, a single-junction solar cell can theoretically emulate a triple-junction solar cell, with its high efficiency. In practice, this goal is far from having been achieved, although the research on this topic is very active worldwide.

The confined states of quantum dots (QDs) may form the IB states [2] alluded to above. IB solar cells [3]-[10] have been made with InAs QDs in a GaAs host. As shown in Fig. 1, the CB offset of the InAs forms a potential well that confines electrons around the QD; these electrons have energy levels which lie in the host bandgap. The QD also produces a VB offset that forms a potential pedestal in the VB which, due to its negative effective mass, also confines electrons in this band. Subbandgap transitions can, therefore, be produced between the confined states in the VB and the CB [11].

For a solar cell engineer, the size of the QDs is of utmost importance. The usual way to obtain this information is to ex-

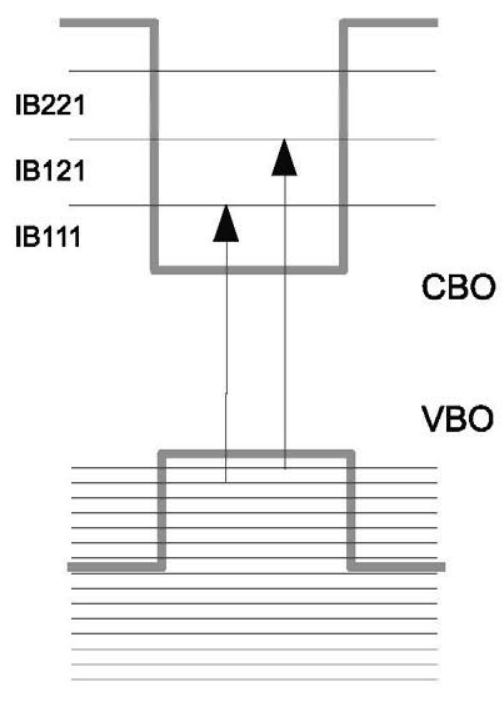

\section{Quantum Dot}

Fig. 1. Conduction band well (CBO) and valence band pedestal (VBO) caused by the band offsets produced by a QD in a host semiconductor, confined levels (horizontal lines), and subbandgap transitions (vertical arrows).

amine cuts of the solar cells by transmission electron microscopy (TEM). This technique is rather sophisticated and has to be entrusted to specialists. Furthermore, the technique is destructive and may not describe well the characteristics on the device in hand. Finally, TEM is accurate to determine the height of the QDs, but it is less accurate in determining the horizontal dimensions of the QDs since the cut may be produced outside their principal axes. Atomic force microscopy may give these dimensions but on structures which are not those in the device and that have suffered different processing.

In this paper, we present a simple procedure for the determination of the horizontal size of the QDs based on the analysis of the quantum efficiency $(\mathrm{QE})$ of the final device. This determination is based on a simplistic model, but the results are adequate.

\section{MODEL}

The first simplification made in the model is on the shape of the QD. Theoretical arguments [12] suggest that in zincblende materials (like all those examined by us), the QDs have the form of quadrangular pyramids that are usually strongly truncated. We approximate this shape as a parallelepiped of square base, this being a similar shape as the one that has been used by 
other scientists [13]-[15]. Other shapes have also been used, like lenses [16] or nontruncated pyramids [17], [18]. The reason for our choice is simplicity.

The second simplification is the use of square offset potentials, shown in Fig. 1, which are often used in the literature. It is well known that strain in the QDs, particularly those grown in the Stranski-Krastanov growth mode, leads to nonsquare potentials with peaks at the QD-host boundary [16]. Such effects are ignored for simplicity.

Strain in the QD also leads to a strong change in the QD bandgap ( 0.418 in the bulk InAs to compare with about $0.740 \mathrm{eV}$ in a QD). This leads to a change in the confining potential of the QD that cannot reasonably be ignored. This has been studied extensively in quantum wells (QWs) by Vurgaftman et al. [19]. However, it is doubtful that results obtained from QWs can be extended to QDs due to the different strain fields. This paper, therefore, offers some information on the QD confining potential based on the QE photoresponse of the finished QD solar cell.

The theory in which our model is based is very simple. It involves the solution of the effective-mass time-independent Schrödinger equation for each band, more specifically for the conduction and the heavy-hole bands. This allows the energy levels of the confined electrons to be obtained in every band, from which peaks to be observed in the QE plot can be calculated. We further consider that the 3-D well is a separable potential so that the 3-D wave function is the product of three 1-D solutions of equations like

$$
-\frac{\hbar^{2}}{2 m_{\mathrm{CB}}} \frac{d^{2} \xi}{d x^{2}}+V_{\mathrm{CBO}}(x) \xi=E_{x} \xi
$$

where $V_{\mathrm{CBO}}$ is the square potential shown in Fig. 1. Bound solutions appear for a finite set of energy values situated inside the well. These solutions, in increasing order, are labeled with a number that becomes a quantum number. In the separable approximation, the 3-D solutions are the product of three functions like $\xi$, one for each variable $(x, y, z)$. Thus, three quantum numbers describe the 3-D wave function. In the 3-D case, the energy of some bound states may exceed the rim of the potential well; these states are called virtual bound states.

Fig. 2(a) shows the spectrum of energies for bound states resulting from the application of this model to the conduction band and to the heavy holes and light holes of the valence band; their quantum numbers are also presented (except for those of the heavy holes, which are too densely packed).

However, even in this simplified model, the separable solution is only an approximation. An exact solution may be found in [20]. Fig. 2(b) shows the energy modifications when a firstorder approximation is used (very close to the exact values). The quantum number labels have been removed by lack of space. It can be observed that the states inside the host material bandgap (the IB states) are accurately calculated with the separable approximation as well as those close to the host bandgap edges. For states deep inside the host semiconductor (GaAs) bands, the differences are visible, but these states play no role in the host subbandgap transitions.

In all these calculations, the effective masses are those of the InAs (where most of the wave function spans). These are

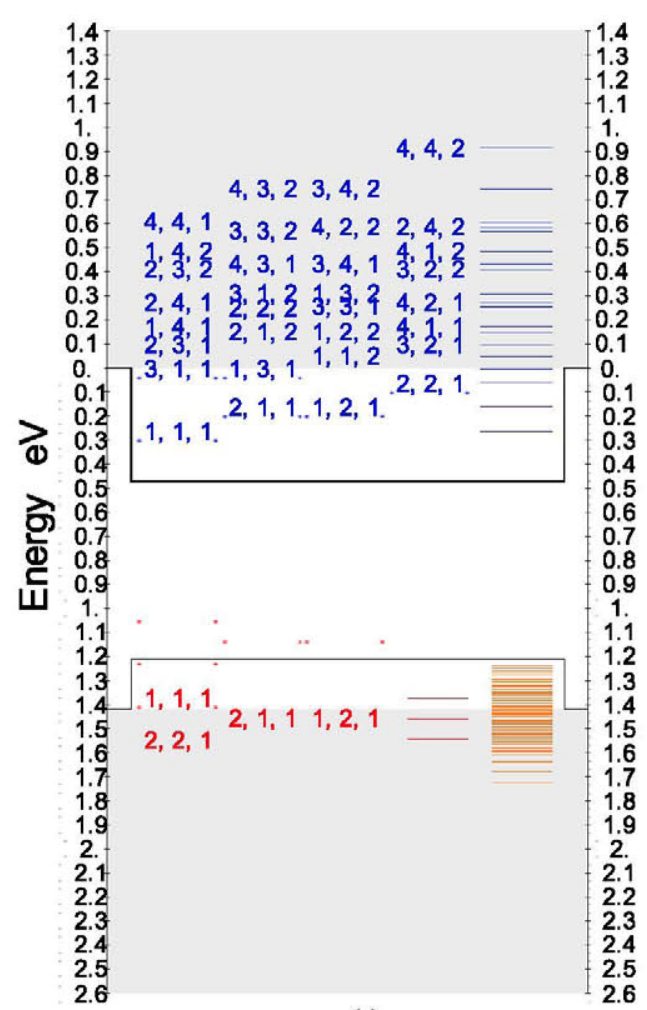

(a)

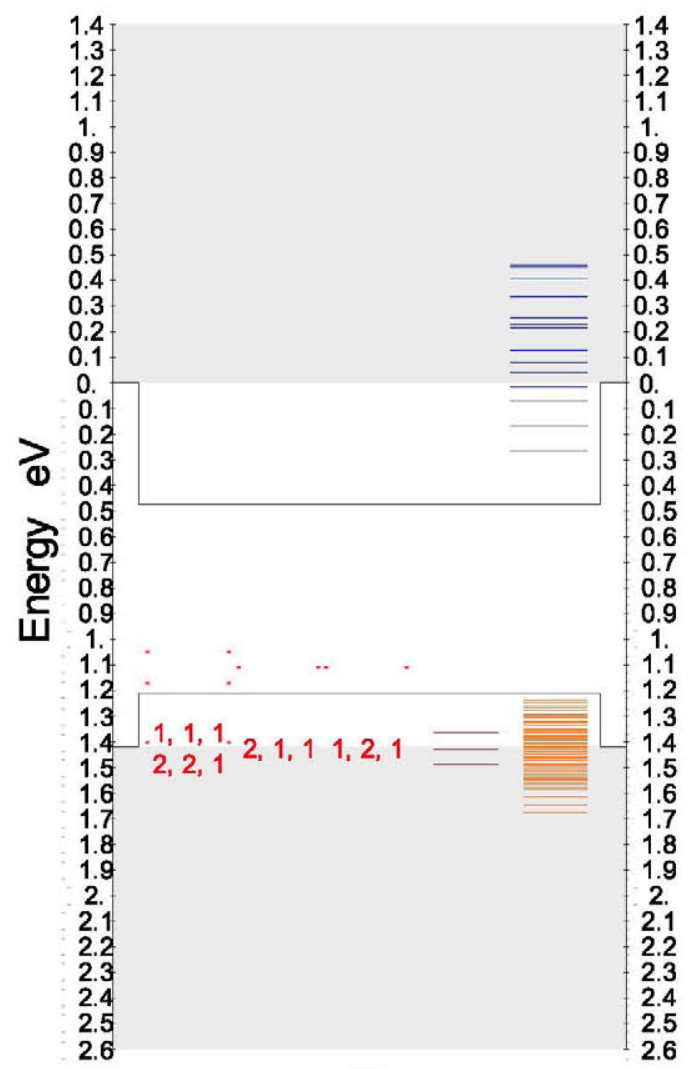

(b)

Fig. 2. (a) InAs/GaAs IB material energy spectrum at the separable approximation. (b) Same energy spectrum at the first-order correction. Blueconduction band states; orange-heavy holes; red-light holes. Quantum numbers are also presented (when space is available). Data as in the line labeled InAs/GaAs of Table I. Gray represents the region of extended states. 
TABLE I

CALCUlation DATA FOR THE ENERGY SPECTRA BASED ON TEM MEASurements and CALCULATIONS IN [21]

\begin{tabular}{lcc}
\hline \hline Sample/Parameter & InAs/GaAs & InAs/Al $_{0.26} \mathrm{Ga}_{0.74} \mathrm{As}$ \\
\hline$m_{\mathrm{CB}} / m_{0}$ & 0.0249 & 0.026 \\
$m_{\mathrm{HH}} / m_{0}$ & 0.333 & 0.333 \\
$m_{\mathrm{LH}} / m_{0}$ & 0.027 & 0.027 \\
$E_{G} / \mathrm{eV}$ & 1.42 & 1.77 \\
$V_{\mathrm{OCB}} / \mathrm{eV}$ & 0.473 & 0.779 \\
$V_{\mathrm{OVB}} / \mathrm{eV}$ & 0.210 & 0.524 \\
height/nm & 6 & 6.6 \\
width/nm & 16 & 20.6 \\
\hline \hline
\end{tabular}

calculated in [21] based on the data in [19]. As already stated, these calculations are intended for QWs and not for QDs, and they differ slightly from those of the homogeneous InAs; thus, we believe they are also approximately valid for the QDs. They appear in Table I.

The offset potentials have also been calculated in [21], but we consider their values less reliable. Finally, the size of the QDs, width, and height are, in a first instance, obtained from TEM pictures. They all appear in Table I as well.

\section{APPLICATION}

Fig. 3 shows the measured [22] internal $Q E$ of prototype solar cells with InAs QDs in a host of GaAs (InAs/GaAs) in (a), (b), and (c) and of another InAs/ $/ \mathrm{Al}_{0.26} \mathrm{Ga}_{0.74}$ As prototype solar cell in (d). There are two clearly visible peaks, labeled $E_{0}$ and $E_{1}$ in (a)-(d).

Some characteristics of the cells measured and the position of the peaks are presented in Table II.

The QE peaks correspond to the transitions $|H H 121\rangle \rightarrow \mid$ IB111 $\rangle$ and $|H H 111\rangle \rightarrow \mid$ IB121 $\rangle$ for the peaks $E_{0}$ and $E_{1}$, respectively, as schematically shown in Fig. 1. $\mathrm{HH}$ stands for heavy holes and IB stands for intermediate band. $|\mathrm{HH} 111\rangle \rightarrow|\mathrm{IB} 111\rangle$ is forbidden by symmetry [24]. The transitions leading to the peaks have been identified in [24] by simulation of the QE. The states $|\mathrm{HH} 121\rangle$ and $|\mathrm{H} H 211\rangle$ are degenerate; both contribute equally to the corresponding peak. The same is to be said of the states $|\mathrm{IB} 121\rangle$ and $|\mathrm{IB} 211\rangle$.

Using the simple model described above, a Wolfram Matematica code has been prepared. Introducing the energies of the two mentioned peaks as input, the values of the width and of $V_{\mathrm{CBO}}$ are obtained immediately (no visible calculation time), if the rest of the parameters are introduced previously (QD material: $m_{\mathrm{CB}}, m_{\mathrm{HH}}, V_{\mathrm{CBO}}$, QD height; host material: $E g$ ). In a variant of the code, the calculated ratio of $V_{\mathrm{CBO}} / V_{\mathrm{VBO}}$ is an input, and in this case, the values of $V_{\mathrm{CBO}}$ and $V_{\mathrm{VBO}}$ are in the output. This is the variant presented in Table III. The outputs are the three last rows.

\section{DISCUSSION}

We can observe that the output sizes are close to the sizes measured by TEM (in Table I). This is a confirmation that the separable model, despite its ideality, can interpret the observed

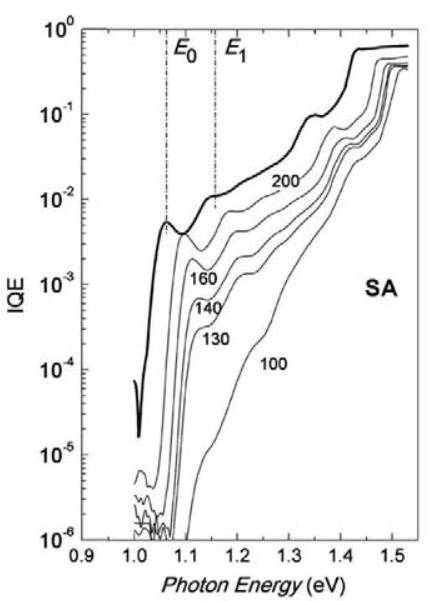

(a)

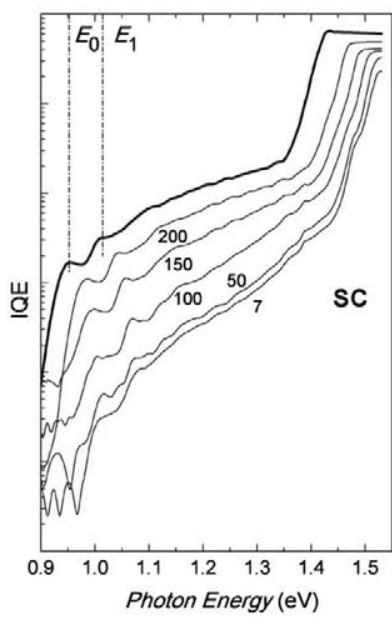

(c)

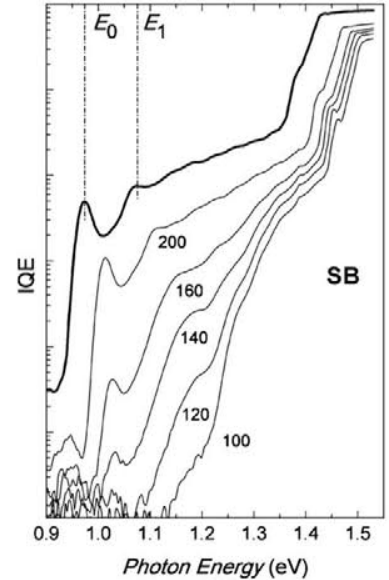

(b)

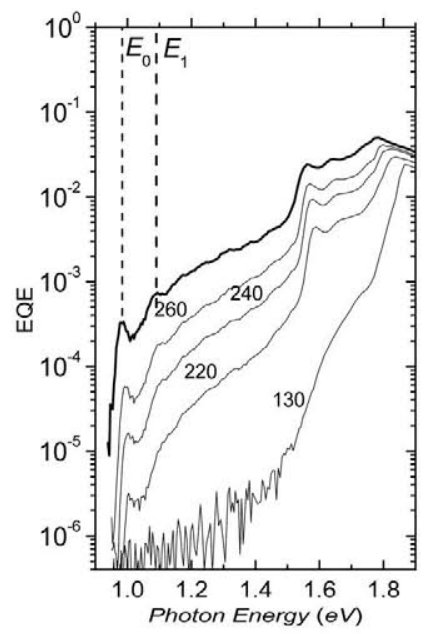

(d)
Fig. 3. IQE of the InAs/GaAs solar cell samples named (a) SA, (b) SB, and (c) SC in [22] (see Table II for information about cell structure) and of the InAs/Al ${ }_{0.26} \mathrm{Ga}_{0.74} \mathrm{As}$ prototype solar cell (d) of [23]. Plots (a)-(c) are reproduced with permission from [22], AIP 2010. Plot (d) is reproduced from [23], IEEE 2012.

TABLE II

SOME CHARACTERISTTCS OF THE SAMPLES PRESENTED IN FtG. 3

\begin{tabular}{lcccc}
\hline \hline Sample & $\begin{array}{c}\text { InAs/GaAs, } \\
\mathrm{SA}\end{array}$ & $\begin{array}{c}\text { InAs/GaAs, } \\
\mathrm{SB}\end{array}$ & $\begin{array}{c}\text { InAs/GaAs, } \\
\mathrm{SC}\end{array}$ & $\begin{array}{c}\mathrm{InAs}_{\mathrm{AAl}} \mathrm{Ga}_{0.74} \mathrm{As} \\
\mathrm{Ga}_{0.74}\end{array}$ \\
\hline QD layers & 50 & 50 & $10+$ seed & 20 \\
InGaAlAs cap & $\mathrm{No}$ & Yes & Yes & No \\
Spacer/nm & 83 & 84 & 13 & 50 \\
$E_{0} / \mathrm{eV}$ & 1.063 & 0.974 & 0.953 & 0.98 \\
$\left(E_{0}-E_{1}\right) / \mathrm{eV}$ & 0.094 & 0.101 & 0.062 & 0.11 \\
\hline \hline
\end{tabular}

data. Second, we see that the size, as interpreted by the model, is usually smaller than the one observed by TEM. This is not surprising: In the cut for the TEM preparation, one may see a diagonal rather than the base side of the QD. Furthermore, the size reported from TEM refers to the largest length, corresponding to the base of the QD, but this size decreases with the height. In any case, the measured size is what the model adopts as the "effective" size of its base for the "equivalent" parallelepiped 
TABLE III

Calculation Data From the QE MEASUREMENTS

\begin{tabular}{lcccc}
\hline \hline Sample & $\begin{array}{c}\text { InAs/GaAs, } \\
\mathrm{SA}\end{array}$ & $\begin{array}{c}\text { InAs/GaAs, } \\
\mathrm{SB}\end{array}$ & $\begin{array}{c}\text { InAs/GaAs, } \\
\mathrm{SC}\end{array}$ & $\begin{array}{c}\mathrm{InAs}_{\mathrm{Ga}} / \mathrm{Al}_{0.26} \\
\mathrm{Ga}_{0} .74 \mathrm{As}\end{array}$ \\
\hline$m_{\mathrm{CB}} / m_{0}$ & 0.0249 & 0.0249 & 0.0249 & 0.026 \\
$m_{\mathrm{HH}} / m_{0}$ & 0.333 & 0.333 & 0.333 & 0.333 \\
$m_{\mathrm{LH}} / m_{0}$ & 0.027 & 0.027 & 0.027 & 0.027 \\
$E_{G} / \mathrm{eV}$ & 1.42 & 1.42 & 1.42 & 1.77 \\
$h_{e i g h t / n m}$ & 6 & 6 & 6 & 6.6 \\
$V_{0 \mathrm{CB}} / \mathrm{eV}$ & 0.418 & 0.491 & 0.476 & 0.638 \\
$V_{0 \mathrm{VB}} / \mathrm{eV}$ & 0.186 & 0.218 & 0.212 & 0.430 \\
width/nm & 15.2 & 14.9 & 20.1 & 15.6 \\
\hline \hline
\end{tabular}

The upper block are parameters in the code; the lower block (last three rows) are outputs. Parameters are from Table I. acterize the QDs. The uncertainties in the offset potentials do not significantly affect these calculations. This determination is obtained in the finished device and is not destructive.

One of the two offsets can also be obtained if the other is known, or both can be calculated if some relationship between them is assumed, for example, keeping the proportion that has been calculated for equivalent QWs.

It would be of interest to apply the simple model used here to other characterization technologies, such as photo- and electroluminescence and photoreflectance measurements. Due to the different excitations modes, this application is not straightforward and requires further study.

\section{REFERENCES}

leading to the observed absorption peaks. It is interesting to see that the sample SC, with fewer layers and a seed below, gives larger dots than the other samples with more layers. The size calculation is also reasonable in InAs/GaAlAs cells of different host material. The same comments are applicable to this case.

The size calculation is more or less independent of the value we set for the height. For cell SB, when introducing 4, 6, and $9 \mathrm{~nm}$, the value of the width is $15.2,14.9$, and $14.6 \mathrm{~nm}$, respectively. Notice that the lattice constant of the unit cells is around $0.6 \mathrm{~nm}$. Therefore, size differences below this value are meaningless. In this framework, the three values calculated above are the same. The model is perfectly applicable to the case of GaAlAs cells with a larger bandgap, giving the same reasonable results. The comments made for the cells in a GaAs host apply to this case as well.

By looking at the output parameters in Table II, we observe that sample SA has a smaller offset than samples SB and SC, both with an InGaAlAs capping (see Table II). Since this capping has a larger bandgap than the InAs itself, it seems to reinforce the confinement and increase the offsets. Thus, the capping is interpreted by the model as a reinforcement of the confining offsets.

The cell with the AlGaAs host shows offsets potentials that are substantially below those calculated in for QWs [21] (see Table I). This behavior is replicated in the SA cell, without capping, in the GaAs host. The strained InAs bandgap is the host bandgap less the two offsets. It seems that the InAs has more strain in the QDs than in the QWs since its bandgap shows a greater increase.

For the same observed peaks, we can calculate $V_{O C B}$ for a given $V_{\mathrm{OVB}}$. This leads, in cell $\mathrm{SB}$, to $V_{\mathrm{OCB}}$ values of 0.613 , 0.499 , and $0.403 \mathrm{eV}$ for $V_{\mathrm{OVB}}$ values of $0.1,0.21$, and $0.3 \mathrm{eV}$, respectively. This behavior is to be expected. The calculated values of the width are now $15.4,14.9$, and $14.3 \mathrm{~nm}$, respectively, all within the $0.6-\mathrm{nm}$ error margin of one unit cell.

\section{CONCLUSION}

In summary, the measurement of the position of the first two peaks of the QE of an IB solar cell with QDs, both clearly visible in most cases, allows us to calculate, with a reasonable accuracy, the base of the equivalent parallelepiped used to char-
[1] A. Luque and A. Martí, "Increasing the efficiency of ideal solar cells by photon induced transitions at intermediate levels," Phys. Rev. Lett., vol. 78, pp. 5014-5017, 1997.

[2] A. Martí, L. Cuadra, and A. Luque, "Quantum dot intermediate band solar cell," in Proc. 28th IEEE Photovoltaics Spec. Conf., 2000, pp. 940-943.

[3] A. Luque, A. Martí, C. Stanley, N. López, L. Cuadra, D. Zhou, and A. Mc-Kee, "General equivalent circuit for intermediate band devices: potentials, currents and electroluminescence," J. Appl. Phys., vol. 96, pp. 903-909, 2004.

[4] S. M. Hubbard, C. D. Cress, C. G. Bailey, R. P. Raffaelle, S. G. Bailey, and D. M. Wilt, "Effect of strain compensation on quantum dot enhanced GaAs solar cells," Appl. Phys. Lett., vol. 92, p. 123512, Mar 2008.

[5] V. Popescu, G. Bester, M. C. Hanna, A. G. Norman, and A. Zunger, "Theoretical and experimental examination of the intermediate-band concept for strain-balanced (In,Ga)As/Ga(As,P) quantum dot solar cells," Phys. Rev. B, vol. 78, p. 205321, 2008.

[6] S. A. Blokhin, A. V. Sakharov, A. M. Nadtochy, A. S. Pauysov, M. V. Maximov, N. N. Ledentsov, A. R. Kovsh, S. S. Mikhrin, V. M. Lantratov, S. A. Mintairov, N. A. Kaluzhniy, and M. Z. Shvarts, "AlGaAs/GaAs photovoltaic cells with an array of InGaAs QDs," Semiconductors, vol. 43, pp. 514-518, 2009.

[7] R. Oshima, A. Takata, and Y. Okada, "Strain-compensated InAs/GaNAs quantum dots for use in high-efficiency solar cells," Appl. Phys. Lett., vol. 93, p. 083111, 2008.

[8] Y. Okada, R. Oshima, and A. Takata, "Characteristics of InAs/GaNAs strain-compensated quantum dot solar cell," J. Appl. Phys., vol. 106, p. 024306 , Jul. 2009.

[9] R. B. Laghumavarapu, M. E1-Emawy, N. Nuntawong, A. Moscho, L. F. Lester, and D. L. Huffaker, "Improved device performance of InAs/GaAs quantum dot solar cells with GaP strain compensation layers," Appl. Phys. Lett., vol. 91, p. 243115, Dec. 2007.

[10] C. G. Bailey, D. V. Forbes, S. J. Polly, Z. S. Bittner, Y. Dai, Chelsea Mackos, R. P. Raffaelle, and S. M. Hubbard, "Open-circuit voltage improvement of InAs/GaAs quantum-dot solar cells using reduced InAs coverage," IEEE J. Photovoltaics, vol. 2, no. 3, pp. 269-275, Jul. 2012.

[11] A. Luque, A. Marti, E. Antolín, P. G. Linares, I. Tobías, I. Ramiro, and E. Hernandez, "New Hamiltonian for a better understanding of the quantum dot intermediate band solar cells," Sol. Energy Mater. Sol. Cells, vol. 95 , pp. 2095-2101, 2011.

[12] D. Bimberg, M. Grundmann, and N. N. Ledentsov, Quantum Dot Hetrostructures. New York, NY, USA: Wiley, 1999.

[13] M. Califano and P. Harrison, "Approximate methods for the solution of quantum wires and dots: Connection rules between pyramidal, cuboidal, and cubic dots," J. Appl. Phys., vol. 86, pp. 5054-5059, Nov. 1999.

[14] O. L. Lazarenkova and A. A. Balandin, "Miniband formation in a quantum dot crystal," J. Appl. Phys., vol. 89, pp. 5509-5515, May 2001.

[15] Q. Shao, A. A. Balandin, A. I. Fedoseyev, and M. Turowski, "Intermediateband solar cells based on quantum dot supracrystals," Appl. Phys. Lett., vol. 91, p. 163503, Oct. 2007

[16] V. Popescu, G. Bester, and A. Zunger, "Coexistence and coupling of zero-dimensional, two-dimensional, and continuum resonances in nanostructures," Phys. Rev. B, vol. 80, p. 045327, Jul. 2009.

[17] C. Pryor, "Eight-band calculations of strained InAs/GaAs quantum dots compared with one-, four-, and six-band approximations," Phys. Rev. B, vol. 57, pp. 7190-7195, Mar. 1998. 
[18] S. Tomic, "Electronic structure of $\operatorname{In}_{\mathrm{y}} \mathrm{Ga}_{1-\mathrm{y}} \mathrm{A} s_{1-\mathrm{x}} \mathrm{N}_{\mathrm{x}} / \mathrm{GaAs}(\mathrm{N})$ quantum dots by ten-band k.p theory," Phys. Rev. B, vol. 73, p. 125348, Mar. 2006.

[19] I. Vurgaftman, J. R. Meyer, and L. R. Ram-Mohan, "Band parameters for III-V compound semiconductors and their alloys," J. Appl. Phys., vol. 89, pp. 5815-5875, Jun. 2001.

[20] A. Luque, A. Mellor, I. Tobías, E. Antolín, P. G. Linares, I. Ramiro, and A. Martí, "Virtual-bound, filamentary and layered states in a box-shaped quantum dot of square potential form the exact numerical solution of the effective mass Schrödinger equation," Physica B, vol. 413, pp. 73-81, 2013.

[21] P. G. Linares, A. Marti, E. Antolin, and A. Luque, " III-V compound semiconductor screening for implementing quantum dot intermediate band solar cells " J. Appl. Phys., vol. 109, p. 014313, 2011.

[22] E. Antolín, A. Marti, C. D. Farmer, P. G. Linares, E. Hernández, A. M. Sánchez, T. Ben, S. I. Molina, C. R. Stanley, and A. Luque, "Reducing carrier escape in the InAs/GaAs quantum dot intermediate band solar cell," J. Appl. Phys., vol. 108, p. 064513, 2010.
[23] I. Ramiro, E. Antolin, M. J. Steer, P. G. Linares, E. Hernandez, I. Artacho, E. Lopez, T. Ben, J. M. Ripalda, S. I. Molina, F. Briones, C. R. Stanley, A. Marti, and A. Luque, "InAs/AlGaAs quantum dot intermediate band solar cells with enlarged sub-bandgaps," in Proc. 38th IEEE Photovoltaic Spec. Conf., New York, NY, USA, 2012, pp. 652-656.

[24] A. Luque, A. Mellor, E. Antolin, P. G. Linares, I. Ramiro, I. Tobias, and A. Marti, "Symmetry considerations in the empirical k.p Hamiltonian for the study of intermediate band solar cells," Sol. Energy Mater. Sol. Cells, vol. 103, pp. 171-183, 2012. 